\title{
Filosofía de la Educación y pedagogía de la enseñanza en la formación del profesorado. Estudio de caso, percepción del estudiantado
}

\author{
Camacho Verdugo, Luis Rodrigo; Morales Paredes, Hernán \\ Filosofía de la Educación y pedagogía de la enseñanza en la formación del profesorado. Estudio de caso, \\ percepción del estudiantado \\ Revista Educación, vol. 44, núm. 1, 2020 \\ Universidad de Costa Rica, Costa Rica \\ Disponible en: http://www.redalyc.org/articulo.oa?id=44060092006 \\ DOI: https://doi.org/10.15517/revedu.v44i1.34179
}

Esta obra está bajo una Licencia Creative Commons Atribución-NoComercial-SinDerivar 3.0 Internacional. 


\section{Filosofía de la Educación y pedagogía de la enseñanza en la formación del profesorado. Estudio de caso, percepción del estudiantado}

\section{Case Study: Student Perception about Philosophy of Education and Pedagogy in Teacher-Training.}

Luis Rodrigo Camacho Verdugo

Universidad Católica de la Santísima Concepción,

Concepción, Chile, Chile

lcamacho@ucsc.cl

Dttp://orcid.org/0000-0003-4480-6543

Hernán Morales Paredes

Universidad Católica de la Santísima Concepción, Chile

hmorales@ucsc.cl

(iD) http://orcid.org/0000-0002-9683-0927
DOI: https://doi.org/10.15517/revedu.v44i1.34179

Redalyc: http://www.redalyc.org/articulo.oa?id=44060092006

Recepción: 25 Marzo 2019

Aprobación: 18 Agosto 2019

\section{Resumen:}

Esta investigación se desarrolla en el contexto educativo de formación del profesorado en la Universidad Católica de la Santísima Concepción, de Chile, tiene como objetivo conocer la percepción del estudiantado de pedagogía con respecto a la asignatura Filosofía de la Educación. La metodología utilizada parte de un diseño descriptivo, cualitativo, a través de un estudio de caso. Se elaboraron entrevistas en profundidad las cuales permitieron construir un relato sobre la percepción y establecer relaciones entre variables asociadas al objetivo. Las respuestas fueron analizadas a través de categorías y se complementó con el uso del software Nvivo 11. Los resultados más relevantes indican que el déficit está asociado a un problema motivacional por parte de los estudiantes hacia el estudio de la filosofía en general. Sin embargo, muestran una percepción positiva hacia la necesaria presencia filosófica en el proyecto curricular para la formación, señalan la necesidad de perfeccionar los recursos didácticos que emplea el personal docente, así como estrategias de enseñanza que ayuden de modo concreto a los estudiantes de pedagogía a desarrollar habilidades reflexivas; se reconoce la necesidad de proponer una didáctica específica de la Filosofía.

Palabras Clave: Filosofía de la Educación, Teoría de la Educación, Didáctica de la Filosofía.

\section{Abstract:}

This study took place in Chile at the Universidad Católica de la Santísima Concepción Faculty of Education. The objective of the study was to gain insight about the perception of Education Majors regarding a Philosophy of Education course. The methodology involved a descriptive, qualitative design presented within a case study based on in-depth interviews. The response through which the authors were able to build a narrative detailing perception and the different variables associated with the objective. Responses were categorized and analyzed using Nvivo 11 software. The most significant results reveal that students are not motivated to take Philosophy of Education or any other type of philosophy course. Yet, they do demonstrate that the study of philosophy is an important part of the Education Major curriculum. More educational resources must be offered, such as additional teaching strategies to help Education Majors further develop their reflexive skills and a renewed didactic approach towards the study of philosophy.

KEYWORDS: Philosophy of Education, Theory of Education, Didactics of Philosophy.

\section{INTRODUCCIÓN}

La enseñanza de la Filosofía se encuentra en un proceso de discusión y análisis en Chile respecto a su relevancia, pues se han presentado opiniones respecto de suprimir la asignatura de filosofía del currículum escolar (Ferrer, 2018). La sola idea de eliminarla ha generado a nivel de la sociedad argumentos que han 
mostrado la importancia de su enseñanza en el sistema escolar, y en opinión de quienes proponen este artículo, además es necesaria una reflexión respecto de qué y cómo enseñar la Filosofía.

En ese sentido es importante considerar que el actual contexto sociocultural de los estudiantes implica la presencia de videojuegos, plataformas web, Facebook, Instagram, YouTube, por nombrar algunos elementos, lo que conlleva a que la enseñanza de la Filosofía debiese incluirlos para el desarrollo de habilidades, conocimientos, competencias, diálogo filosófico. Para esto se requiere que el profesorado posea una didáctica de la Filosofía, adecuada a los contenidos propuestos por el currículum escolar, y que considere elementos del contexto del estudiantado.

En este contexto se plantea el objetivo de este trabajo, el cual es dar cuenta y analizar cualitativamente la percepción que tienen los estudiantes de pedagogía sobre la Filosofía de la Educación, en un proceso de formación del profesorado en cuyo currículum se encuentra presente la reflexión filosófica, y en el que el estudiantado forma parte de este proceso de aprendizaje y enseñanza académica.

\section{ANTECEDENTES DEL PRoblema}

En la formación del profesor(a) en la Universidad Católica de la Santísima Concepción (UCSC, 2016), la asignatura de Filosofía de la Educación está presente en el currículo de las carreras de pedagogía, y se ubica dentro del área de la formación profesional pedagógica, en este contexto la investigación se plantea la preocupación respecto al desarrollo y logro del objetivo de esta asignatura, de modo que cumpla con los fines ontológicos que la definen; para ello se ha propuesto en este trabajo determinar la percepción de los estudiantes de pedagogía respecto de la Filosofía de la Educación, es decir, desde sus puntos de vista, para así enriquecer un análisis y discusión sobre los aspectos que debe considerar esta asignatura en la formación.

Existen varias razones que justifican el desarrollo de esta investigación, en primer lugar en la revisión bibliográfica, se han encontrado estudios o investigaciones sobre la percepción con respecto a las clases de filosofía en general, por ejemplo: Moreno (2013) muestra una investigación hacia la clase de filosofía en la Universidad Nacional Autónoma de Honduras; Sarbach (2005) ¿Qué pasa en la clase de Filosofía?; Carvajal y García (2004), cómo perciben los estudiantes universitarios la enseñanza de la Filosofía en Costa Rica; Cárdenas (2007), que muestra metodologías de la enseñanza de la Filosofía en la educación media chilena. Sin embargo, con respecto al estudio sobre el desarrollo y logros de los objetivos de la asignatura filosofía de la Educación en quienes se están formando como docentes, hasta el momento las personas investigadoras no han encontrado antecedentes. Segundo, la necesidad de obtener datos precisos de la opinión del estudiantado en el ámbito señalado para la toma de decisiones en aspectos curriculares administrativos de la formación del profesorado en la UCSC, como por ejemplo la renovación que considera una modificación de un currículum por objetivos a uno por competencias, la que se explica por lo dinámico de un proceso de formación. Tercero, con esta investigación se espera contribuir en el análisis del perfil de egreso de un modo más sistemático, pues una de sus orientaciones es un dominio del área principal de conocimiento científico y profesional, el cual incluye la dimensión filosófica por ser un fundamento de la pedagogía tanto como disciplina, y como ejercicio personal. Por lo tanto, sí existen disminuidos logros de los objetivos de aprendizaje -o en uno de ellos- en la asignatura filosófica, esto podría afectar directamente al perfil de egreso que refiere, tal como se manifiesta en el Modelo Educativo de la UCSC, a formar un profesional de la educación que desarrolle las máximas potencialidades personales, éticas, valóricas y profesionales que se espera que logren al concluir su etapa académica. Como cuarto argumento, se espera contribuir a irradiar de modo más notorio la centralidad de la persona humana, su dignidad y su ética; esto es posible ya que la Filosofía de la Educación es una disciplina que fortalece el diálogo con las ciencias humanas y responde sus inquietudes; por ende, si se considera esta afirmación se podrá solucionar la dificultad encontrada, a saber, se podrá ayudar directamente a que no tan solo logren los objetivos de la asignatura respectiva, sino que además las enriquecerá como personas y ayudará a que vean sus futuros estudiantes con una sensibilidad mayor, desde un punto de vista holístico o 
integral; este aspecto cobra mayor realce en relación con el campo laboral en que tendrán que desempeñarse. Finalmente, la quinta razón, es que la existencia de la Filosofía de la Educación en el currículum de formación favorece a una mayor vinculación entre el estudiantado y los planteamientos públicos en los cuales tendrá que desenvolverse el nuevo profesional. En este sentido, se puede agregar que, si tienen una mejor preparación filosófica, la elaboración de su saber pedagógico estará mejor consolidada y argumentada, lo cual resulta relevante cuando deben manifestar opiniones relativas a cuestiones valóricas o actitudinales de la sociedad y la cultura escolar en su desempeño cotidiano.

\section{2.- Referente teórico:}

\section{1. ¿Es necesaria la Filosofía?}

En estos días existe un ambiente que se centra básicamente en lo pragmático, en lo que produce utilidad. De hecho, algunos piensan que la Filosofía es inútil (Cerletti, 2008) a tal punto que en países como España y México se evidencia un desplazamiento de ella de los sistemas educativos (Muñoz, 2015); en el caso de Chile, la Filosofía tiende a ser incorporada dentro de una temática psicológica (Olivares, 2015). Por ende, es evidente alguna vez preguntarse sobre si esta actitud o perspectiva que se tiene sobre la Filosofía o de tener alguna asignatura filosófica en el proceso formativo de un profesional es errónea y por qué. Para dar una respuesta a esta interrogante se debe considerar que la palabra utilidad puede tener distintos significados; primero, en el contexto de una sociedad de mercado en donde todo se centra en producción y reproducción, la Filosofía se puede tratar de encasillarla en una mercancía, en donde todo se reduce a lo empírico; en otras palabras, si ella no incide de modo directo en algo concreto, entonces no me sirve. Por ejemplo: ¿̨si curso filosofía tendré mayor posibilidad de ingreso económico? La respuesta negativa llevaría a decir entonces que no es necesaria. Por lo tanto, no es de extrañar que toda perspectiva tecnocrática combata a la Filosofía de modo arduo puesto que no le encuentra sentido (Cortés, 2015). El segundo significado, referiría en mostrar a la Filosofía como una especialidad para la argumentación, lo cual la reduciría a presentar simplemente las opiniones o relaciones establecidas para lograr algo específico y concreto, por ejemplo: como asesor de empresa, o una temática de la psicología; en este punto tampoco sería necesaria la Filosofía, pues no se podría distinguir con otra profesión, o incluso estaría en desventaja, por el supuesto erróneo de que la Filosofía no tiene sistematización epistemológica suficiente (Olivares, 2015). El tercer significado, brota de la naturaleza de la Filosofía, la cual busca que se logre un diálogo crítico y transformador que, por una parte, analice los objetivos que constituyen a la sociedad, y por otra, profundice sobre el modo de concebir el mundo y su sentido último. Por lo tanto, este sentido se desvincula de los dos anteriores. (Azar, 2015). De ahí que la Filosofía, desde una perspectiva transversal, se entienda como una participación humana de la sabiduría ideal, en donde el concepto sabiduría ideal integra los distintos matices que tiene esta actividad, los cuales se expresan en el esfuerzo humano de potenciar al máximo el saber racional y, al mismo tiempo, lograr un saber totalizante que incluya los distintos saberes de las ciencias particulares, en especial el saber educativo y pedagógico (Millán, 2013; Martínez, 2011; González, 1963). Por lo tanto, la Filosofía siempre será necesaria, pues potencia al ser humano a su perfección. Si se niega su necesidad, se autodestruye, quedando en el error y obstaculizando cualquier posibilidad de salir de ello.

\subsection{La enseñanza y el aprendizaje de la Filosofía}

El tema sobre la importancia del correcto aprendizaje de la Filosofía también ha crecido en notoriedad en el último tiempo. Roberto Miguel Azar (2015) afirma que, en el proyecto de educación integral de la persona humana, la Filosofía no puede ser reducida a una mera repetición de contenido, sino que a distintos elementos 
que permitan realmente suscitar el aprendizaje. Además, se destaca que ese mismo aprendizaje permite que el estudiantado universitario desarrolle aspectos actitudinales como ser mejores personas tal como lo afirma Kohan (2010):

La filosofía como ciencia, propone un instrumento teórico para poder ser crítico y propositivo, intenta superar las apariencias de la realidad -inclusive la de los datos verificables-, a Dios, a la historia, a la psique, a la bondad, etc.... ¿Qué pretende enseñarnos la Filosofía?, Ante todo que somos humanos, y por ende cómo podemos ser mejores personas, también nos propone la posibilidad de elaborar un proyecto de vida de acuerdo con nuestra vocación y con una direccionalidad de cara a mejorar la historia; nos enseña a vivir, no como datos o cifras, sino como un ser de valores que puede dar más de sí; nos abre los ojos para superar las apariencias y para cuestionar el dogmatismo rígido (p. 30).

El aprendizaje de la Filosofía establece un vínculo entre el cuerpo docente y el estudiantado para que de esa manera desarrollen su propio pensamiento y pueda adoptar una cierta actitud frente a la vida (Azar, 2015; Martínez 2011). En otras palabras, la enseñanza de la Filosofía posibilita el aprendizaje y articulación de saberes que, a partir de la investigación, hace posible la marcha de ciertos aspectos intelectuales propios y la adquisición de otros para comprender los objetos de estudio, lo que favorecerá el actuar sobre la realidad en su conjunto o en parte de ser necesario (Boavida, 2006; Fullat 1992). En este aspecto, cobra relevancia entonces en el trabajo docente el qué y cómo enseñar, y cómo el alumnado aprende. Estos aspectos sin duda deben ser promovidos para que el mismo estudiantado se responsabilice de su propio aprendizaje. De aquí que se exprese la importancia de una didáctica de la Filosofía pertinente para el actual escenario social, pues la Filosofía puede aportar en el nivel educativo de formación profesional a encontrar la forma adecuada de responder a los cambios que ocurren en el mundo actual, los que además, son constantes y acelerados, por lo tanto, preguntas como ¿qué tipo de ser humano formar? ¿qué saberes y habilidades se debe promover para los ámbitos personal, social y laboral?, nunca perderán vigencia (Cortés, 2015; Cerletti 2008).

Ahora bien, es importante destacar que la importancia del aprendizaje y de la enseñanza de la Filosofía en la Educación Superior no es una motivación o interés de un grupo pequeño o de un par de personas, sino que tiene un gran reconocimiento internacional. Se destaca como ejemplo la publicación de UNESCO (2011), en la cual se dedica el tercer capítulo a investigar sobre la importancia de enseñar y aprender filosofía en el ámbito universitario.

Además, hay investigaciones que entregan orientaciones para avanzar en la presencia de una didáctica de la Filosofía. Bernal (2015) ratifica la siguiente propuesta de educación filosófica para llevar a la práctica: ser democrática, es decir, que nadie puede ser excluido de la Filosofía; transdisciplinario, debe tener un contacto y diálogo con las otras áreas del conocimiento; aprendizaje por sus causas, a partir de los primeros y principales conceptos para poder tener una elaboración más sistemática; carácter intercultural, pues debe haber un intercambio entre culturas y diversas sociedades; la incorporación de nuevas herramientas de enseñanza, acá se refiere a la inclusión de las nuevas tecnologías (TIC).

Por otra parte, Moreno (2013) entrega información sobre factores que pueden influir en que el aprendizaje de los estudiantes se vea dificultado, por ejemplo:

se le ha reprochado con frecuencia a la Filosofía de ser un lujo, un pasatiempo o un saber que no sirve para nada. Hay quizás en estas afirmaciones el estigma venido de malos maestros practicantes de la clase de Filosofía, de la poca formación en la disciplina o de la no comprensión de la Filosofía como saber y reflexión fundamental sobre el mismo hombre. (p. 29).

Más adelante, el mismo autor expresa:

podemos interpretar que los estudiantes perciben un nivel de aridez temática y manifiestan la urgencia de una didáctica que permita comprender con mayor profundidad, tanto las corrientes filosóficas, como los postulados que se enseñan como parte de los requerimientos básicos de la asignatura. (p. 34)

Así se alude a considerar que en el trabajo docente se propicie que el estudiantado efectúe una conexión entre el contenido filosófico que se aprende y aspectos de la vida cotidiana (Azar, 2015; Vásquez 2012), y 
se espera reconocer que hay docentes que tienen conciencia de la importancia sobre el modo de enseñar la Filosofía y su pertinencia (Donda, 2015).

\subsection{La Filosofía en la educación}

Durante la historia de la humanidad la educación siempre ha estado presente y se le ha vinculado al ser humano, de ahí que a medida que avance el tiempo surjan diversas reflexiones sistemáticas o estudios científicos sobre su existencia y finalidad; En este punto, la Filosofía no ha estado al margen de ello. Por señalar algunos autores -de modo breve-, en la antigüedad se destaca a Sócrates, el cual expresa que la mayéutica es una instancia que favorece el aprendizaje, pues por medio de una reflexión personal el individuo, mediante la ayuda de un apoyo o docente, puede llegar a conocer la verdad. También se encuentra a Platón (427-347 a. e. c.), quien afirma en su obra la República que el ciudadano debe cumplir un perfil en donde la educación obliga a saber más que el común; y a Aristóteles (384-322 a. e. c.), cuya idea central gira en que la educación es algo natural en el ser humano y esta comprende lo que es mejor al ser humano, en especial a través de las reglas civiles y morales (Camacho, 2017).

En la Edad Media, se destaca a Santo Tomás de Aquino (1225-1274), que en referencia a la educación hace una validación de los padres en la tarea educativa y destaca el carácter formativo de la educación que concede al ser humano crecer en la perfección (Millán, 2013). En la modernidad, en donde se empieza a destacar el carácter práctico de la educación, se encuentra John Locke (1632-1704) su aporte filosófico sobre la educación se centra en que la educación debe estar en armonía con la socialización de la clase; y Johann Herbart (1776-1841), afirma que la educación tiene por base la instrucción; por ende, deben poseer personalidad y carisma para promover el aprendizaje. En la época contemporánea, se encuentra John Dewey (1859-1952) cuya filosofía educativa se centra en dos ideas, la primera, la educación es como un proceso para formar disposiciones fundamentales respecto a los seres humanos; y segunda, la dimensión educativa es como una técnica, pues es una reconstrucción de la experiencia -o una reorganización de esta- que le da sentido a la experiencia y que aumenta la capacidad de poder dirigir los aspectos que se desprenden de ella. Otro autor de esta época, Richard Stanley Peters (1919-2011) afirma que la educación debe girar en torno a los objetivos de especificar con la mayor exactitud posible lo que se quiere hacer y que la educación consiste en aprender algo de valor que comporte un conocimiento organizado el cual el educando adquiere para sí (Camacho, 2017; Altarejos y Naval, 2011).

Por último, en la actualidad la Filosofía sigue siendo un aporte en el ámbito educativo, de hecho, no hay educador que en más de alguna vez se ha hecho la pregunta ¿Cómo educar? ¿Cómo cumplir con los objetivos esperados? Esto refleja que el educador tiene conciencia y sienten el peso de la responsabilidad que tiene su labor; por eso, la pregunta ¿Cómo educar? Y a partir de ahí responde con una mirada filosófica específica. Ahora, eso no quita que esta interrogante sea compleja por ello, para responderla adecuadamente, no solo hay que mirarla desde la Filosofía, sino que además de modo integral, es decir, en sus diferentes dimensiones. De esta integridad se ocupa las Ciencias de la Educación, y en el campo de la formación de un futuro profesorado se trata de plasmar este esfuerzo, por ende el estudiantado desde sus inicios en la formación se nutre de diferentes ciencias, entre ellas la Filosofía Educativa, para poder tener una noción más acabada de lo que es la educación y de lo que significa para el ser humano (Chávez, 2011; Vásquez 2012; Fullat, 1992).

\subsection{La Filosofía de la Educación}

Actualmente existen variados desafíos y exigencias hacia el ámbito educativo, que provienen de los cambiantes procesos sociales, políticos, culturales de la sociedad. Se espera que el profesorado de filosofía tenga las habilidades necesarias para responder desde su profesión a esas exigencias, y que los procesos de 
formación que se desarrollan en las instituciones de Educación Superior (García y Monzón, 2015) consideren formar desde una visión amplia de paradigmas y determinismos, el desarrollo de un pensamiento y diálogo reflexivo que genere espacios formativos para favorecer una construcción comunitaria del conocimiento, la aceptación de la complejidad y el encuentro con los otros. También mantener la relación teoría y praxis en el ámbito educativo (Álvarez, 2012). Ahora bien, la Filosofía es la disciplina por excelencia que contribuye a hacer posible estos fines (García y Monzón, 2015), de ahí que se le llame Filosofía de la Educación (García y Monzón, 2015; Martínez, 2011; González, 1963).

La filosofía educativa nace de la reflexión de los ámbitos teórico-práctico de la pedagogía, el cual es fruto de la acción del ser humano, es decir, de la acción de educar surge una realidad nueva (Vásquez, 2012); y como ya se expresó, esta reflexión se ha hecho desde tiempos remotos. Sin embargo, como disciplina epistemológica prácticamente se ha ido constituyendo a partir del siglo XVIII (Camacho, 2017); y en cuanto disciplina académica, o asignatura es bastante joven (Altarejos y Naval 2011).

Ahora bien, la importancia de aprender Filosofía de la Educación plantea el desafío de tener una visión más amplia que la reducción del ser humano que postula la mentalidad moderna, y por ende no puede ser excluida de la malla curricular tal como lo afirman Vásquez (2012), Martínez (2011) y González (1963).

Esta visión más amplia de la Filosofía de la Educación viene dada por su carácter reflexivo sistemático, la cual se sustenta en la estrecha vinculación que tiene con la pedagogía, antropología y ética,

la Filosofía educativa tiene una relación de necesidad con la pedagogía, pues reflexiona de modo sistemático y bajo la perspectiva de conjunto, la cual incluye una visión antropológica, ética, política, etc., sobre el sentido de la educación, y así marca la dirección humana de los descubrimientos que se realizan en esta ciencia práctica (Camacho, 2015, pp. 64-65).

Por lo tanto, la Filosofía de la Educación ayuda a tener una visión transversal del ámbito educacional, y por ende, por su contenido y su aspecto teórico-práctico, es una importante contribución al logro de la competencia de formación que señala Campos (2012) referida a la comprensión del fenómeno de la diversidad humana como para situar su quehacer profesional en el contexto de las necesidades educativas especiales.

En síntesis, para responder a la pregunta ¿para qué aprender Filosofía de la Educación en el proceso formativo del cuerpo docente?, esta propuesta consiste en que sirve para reflexionar sobre la acción de educar y a partir de ahí orientar y proponer elementos que consoliden el saber educativo y pedagógico, mediante la visión unitaria o totalizante de las distintas disciplinas que conforman las Ciencias de la Educación. Sin duda que esta respuesta no es tan simple llevarla a la práctica, pues la Filosofía educativa tiene múltiples vertientes y esto influye en el proceso formativo, por ejemplo: Filosofía educativa kantiana, Analítica, Teoría de la educación; así como su relación con las Ciencias de la Educación también es compleja, algunos relacionan la Filosofía de la Educación con la Pedagogía -por ejemplo: la ideología alemana-, otros reducen -por ejemplo: la tradición positivista francesa- la Filosofía de la Educación a Sociología educativa. Sin embargo, esto no tan solo muestra la riqueza de la Filosofía, sino que también los desafíos para hacer orientaciones y propuestas educativas lo más objetivas posible (Vásquez, 2012).

\section{Antecedentes Metodológicos}

\section{1.- Participantes}

Este trabajo se elaboró con 18 estudiantes de Pedagogía en educación diferencial, de ler año de formación, cohorte 2016. Se consideró la facilidad de acceso a la muestra, pues el investigador principal es docente de la asignatura y que la asignatura de Filosofía de la Educación, al pertenecer al área de la formación profesional pedagógica, presenta los mismos objetivos para todas las carreras de pedagogía. Al estudiantado se le solicitó de modo voluntario poder ser entrevistado de modo presencial en una de las dependencias de la Facultad de 
Educación de la UCSC. En el momento de efectuar la actividad programada se les notificó que el audio de sus respuestas iba a ser grabado, y se les garantizó el anonimato, el alumnado firmó un consentimiento.

\section{2.- Diseño del estudio}

El diseño se basó en un método descriptivo, cualitativo (Lamoureux, 2006; Rodríguez, Gil y García, 1999), el cual permitió construir un relato detallado sobre un fenómeno, y establecer relaciones entre variables asociadas al objetivo, utilizando una muestra no representativa de la población docente en formación. El método de entrevista se observó apropiado para obtener información de los participantes, pues representan de toda la formación del profesorado en la UCSC, al ser la Filosofía de la Educación una asignatura transversal a todas las carreras de pedagogía. Para ello se ejecutó una entrevista que permitió obtener información de naturaleza privada y anónima a través de preguntas a cada participante respecto de la percepción de la Filosofía de la Educación. A partir de los elementos del marco teórico se construyeron categorías orientadoras para la construcción de las preguntas, que se muestran en la Tabla 1. Estas categorías son la matriz de la cual se procede a elaborar las preguntas que se le aplicarán al estudiantado.

TABLA 1

Categorías orientadoras para la elaboración de preguntas

\begin{tabular}{ll}
\hline Categoria & Definición de la categoría \\
\hline $\begin{array}{l}\text { Gestión académica y didáctica } \\
\text { del profesorado }\end{array}$ & $\begin{array}{l}\text { Los métodos que utiliza el } \\
\text { profesor(a) para enseñar }\end{array}$ \\
\hline $\begin{array}{l}\text { Motivación en el estudiantado } \\
\text { para aprender el contenido de }\end{array}$ & $\begin{array}{l}\text { Los beneficios y dificultades } \\
\text { que tienen para aprender la } \\
\text { la asignatura de Filosofía de la } \\
\text { Educación }\end{array}$ \\
\hline $\begin{array}{l}\text { La existencia de una relación } \\
\text { adecuada entre teoría y praxis } \\
\text { en el proceso académico }\end{array}$ & $\begin{array}{l}\text { La importancia de la } \\
\text { correra }\end{array}$ \\
\hline
\end{tabular}

Fuente: Elaboración propia

La entrevista consideró las características de ser transversal (observación del estado actual), situacional (estudio desde los que han participado, formal (preparada previamente), semiestructurada, y se aplicó una única vez al inicio de la primera clase de la asignatura de Filosofía de la Educación. Se elaboraron 10 preguntas que fueron sometidas a validación a través de juicio de expertos y que se presentan en la Tabla 2. Estas preguntas aplicadas al estudiantado se relacionan con las categorías orientadoras establecidas. 
TABLA 2

Estructuración de preguntas para la entrevista semiestructurada

\begin{tabular}{|c|c|}
\hline $\begin{array}{l}\text { Definición } \\
\text { de la } \\
\text { categoria }\end{array}$ & Preguntas \\
\hline $\begin{array}{l}\text { Los } \\
\text { métodos } \\
\text { que utiliza } \\
\text { el profesor } \\
\text { o la } \\
\text { profesora } \\
\text { para } \\
\text { enseñar }\end{array}$ & $\begin{array}{l}1 \text { ¿Qué métodos utiliza en general el profesor(a) para la } \\
\text { clase y que podrian ser efectivos para una buena } \\
\text { enseñanza de la asignatura en cuestión? } 2 \text { ¿Qué } \\
\text { expectativas tienes con respecto al profesor(a) que } \\
\text { realizará la asignatura filosófica durante el segundo } \\
\text { semestre? }\end{array}$ \\
\hline $\begin{array}{l}\text { Los } \\
\text { beneficios } \\
\text { y } \\
\text { dificultades } \\
\text { que tienen } \\
\text { para } \\
\text { aprender la } \\
\text { asignatura } \\
\text { filosófica }\end{array}$ & $\begin{array}{l}\text { ¿Qué dificultades tienes para aprender en la clase de } \\
\text { filosofía educativa? ¿Cuáles son tus métodos para } \\
\text { aprender la asignatura filosófica? ¿Cuáles son tus } \\
\text { motivaciones para estudiar esta asignatura? }\end{array}$ \\
\hline $\begin{array}{l}\text { La } \\
\text { importancia } \\
\text { de la } \\
\text { asignatura } \\
\text { filosófica } \\
\text { para la } \\
\text { carrera }\end{array}$ & $\begin{array}{l}\text { ¿Cuáles crees que son los motivos para continuar con } \\
\text { esta asignatura en la malla de la carrera? ¿Qué aportes } \\
\text { te entrega esta asignatura para superar los conflictos } \\
\text { educacionales actuales? ¿Cuáles son los beneficios de } \\
\text { tener esta asignatura para tu desenvolvimiento } \\
\text { profesional? }\end{array}$ \\
\hline
\end{tabular}

Fuente: Elaboración propia

Posteriormente se hizo una invitación voluntaria a los 33 estudiantes que forman el curso de los cuales accedieron a ser entrevistados 18 estudiantes; luego se procedió a la transcripción digital de las grabaciones de ellas. Con este material se hizo uso del software de análisis Nvivo 11 para los procesos de análisis descriptivos y las codificaciones respectivas.

\section{4.- Resultados y SUS ANÁlisis}

A partir de la información que se obtuvo de la entrevista se procede a analizarlas con el objetivo de conocer la percepción del estudiantado sobre la enseñanza de la Filosofía de la Educación en la formación del profesorado, y se establecen las categorías respectivas. Con esta información se lleva a cabo un análisis de conglomerados por referencia de palabras mediante el coeficiente de Jaccard de todas las entrevistas, luego se procede a consolidar la obtención adecuada de las categorías; establecer una jerarquía de categorías, y elaboración de un mapa conceptual que exprese los resultados obtenidos. La Tabla 3 muestra las categorías establecidas en tres niveles y su codificación: 
TABLA 3

Categorías y codificación

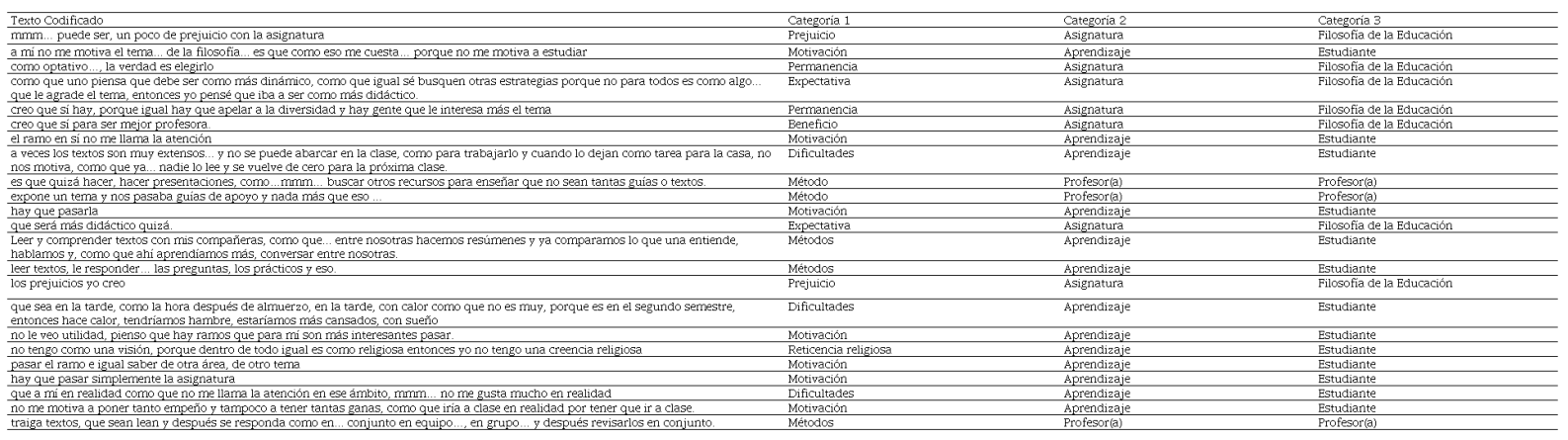

Fuente: Elaboración propia

En el primer análisis, en referencia a la percepción del estudiantado, el cual expresa aspectos interesantes a considerar. El primer texto codificado señala "puede ser, un poco de prejuicio con la asignatura" (estudiante 1, comunicación personal, 11 de agosto de 2017). De este aporte se hace notar algo que es prácticamente evidente, a saber, prejuicios sobre la asignatura de Filosofía; esto no es exclusivo de la realidad chilena, sino que se extiende más allá de ella. Si bien puede haber prejuicios positivos, fácilmente se observan los negativos. En algunos institutos educacionales se ha eliminado la asignatura filosófica y varias veces ha corrido peligro de desaparecer de la enseñanza media chilena. También se encuentra otro texto a destacar, "como que uno piensa que debe ser como más dinámico, como que igual sé busquen otras estrategias porque no para todos es como algo... que le agrade el tema, entonces yo pensé que iba a ser como más didáctico" (estudiante 2, comunicación personal, 11 de agosto de 2017). De esta respuesta se puede deducir la importancia de una actualización en la estrategia didáctica de la Filosofía, que sin duda ayude al contexto moderno, el cual se caracteriza por un entorno tecnológico. A ello hay que precisar que, esta estrategia de didáctica de la Filosofía debe favorecer un aprendizaje para los estudiantes, en el que no solo se incluya un aspecto conceptual, sino que además favorezca un aprendizaje más participativo y significativo; y una disminución del desagrado por la asignatura de filosofía.

En otro de los textos codificados se encuentra lo siguiente: "creo que sí hay, porque igual hay que apelar a la diversidad y hay gente que le interesa más el tema" (estudiante 3, comunicación personal, 11 de agosto de 2017), esta afirmación es la respuesta a la pregunta que refiere sobre la permanencia de la asignatura filosófica en la malla de la asignatura (ver Tabla 2). Lo interesante de esto es que efectivamente se ve una concepción de que la Filosofía debe estar presente ya que esto es una expresión o demostración de aceptar la diversidad de pensamientos, en otras palabras, hay una consideración hacia los estudiantes que deseen estudiar alguna asignatura filosófica. Otro aspecto que se desprende de esta respuesta es que, a pesar de haber un ambiente general que está contra el interés por la Filosofía, siguen existiendo personas que consideran que debe permanecer en el proceso de aprendizaje del estudiantado de educación superior. 
(...) a veces los textos son muy extensos... y no se puede abarcar en la clase, como para trabajarlo y cuando lo dejan como tarea para la casa, no nos motiva, como que ya nadie lo lee y se vuelve de cero para la próxima clase (estudiante 4, comunicación personal, 11 de agosto de 2017).

Acá llama la atención el cierto desgano respecto al uso del texto filosófico que tiene el o la estudiante, lo que puede dar paso a preguntas como: ¿El o la estudiante trae bajas habilidades previas? ¿Los textos serán demasiados complejos para un estudiante de educación superior? ¿Los métodos didácticos del docente serán los adecuados? Independiente de las preguntas que pudiesen seguir apareciendo o las respuestas que pudiesen surgir, lo cierto es que el o la estudiante encuentra un cierto obstáculo que lo afecta en la motivación y que trae como consecuencia que llegue poco preparado a la siguiente clase, lo que sin duda propiciaría que las dificultades del aprendizaje de la Filosofía sean mayores y se forme una apreciación negativa sobre la asignatura. En la última respuesta que se encuentra en la Tabla 3 se puede leer lo siguiente: "traiga textos, que lean y después se responda como en... conjunto en equipo..., en grupo... y después revisarlos en conjunto" (estudiante 5, comunicación personal, 11 de agosto de 2017). El aporte aquí entregado, permite que se interprete la necesidad de que el profesorado utilice una didáctica adecuada que ayude a poder adentrarse a un análisis propicio; en este contexto estaría sugiriendo que, así como hay una etapa de lectura individual, también se usará un espacio de interacción grupal para que aquel que tuvo mayores dificultades, pueda ser ayudado por otro en la lectura del texto filosófico.

Luego se desarrolla el análisis cualitativo descriptivo de los datos obtenidos. Este proceso se inicia con la determinación de ciertas categorías que mostrarán cómo se comienza a levantar la información que se desea obtener. La consolidación de las categorías en las entrevistas se presenta en la Tabla 4 y corresponden al análisis de las repuestas del estudiantado y para su fundamentación se procede a considerar la cantidad de veces que refieren a lo identificado.

TABLA 4

Número de referencias por categoría

\begin{tabular}{lll}
\hline Categoria & $\begin{array}{l}\text { Número de } \\
\text { referencias. } \\
\text { Entrevista 1 }\end{array}$ & $\begin{array}{l}\text { Número de } \\
\text { referencias. } \\
\text { Entrevista 2 }\end{array}$ \\
\hline Estudiante & 7 & 6 \\
\hline Aprendizaje & 7 & 6 \\
\hline Filosofía de la Educación & 6 & 1 \\
\hline Asignatura & 6 & 1 \\
\hline Motivación & 3 & 4 \\
\hline Existencia de prejuicios & 2 & 0 \\
\hline Obstáculos & 2 & 1 \\
\hline Permanencia. & 2 & 0 \\
\hline Profesor(a) & 1 & 2 \\
\hline Métodos del y de la & 1 & 2 \\
estudiante & & \\
\hline Reticencia religiosa & 1 & 0 \\
\hline Métodos del profesor(a) & 1 & 1 \\
\hline Expectativas & 1 & 1 \\
\hline Beneficio & 1 & 0 \\
\hline
\end{tabular}

Fuente: Elaboración propia

Con esta información se procede a desarrollar un análisis de conglomerados por referencia de palabras mediante el coeficiente de Jaccard que presenta la cercanía o lejanía de los conceptos entre sí, y que se muestra en la Figura 1, de las dos entrevistas en conjunto, con ello se procede a consolidar la obtención adecuada de las categorías: 


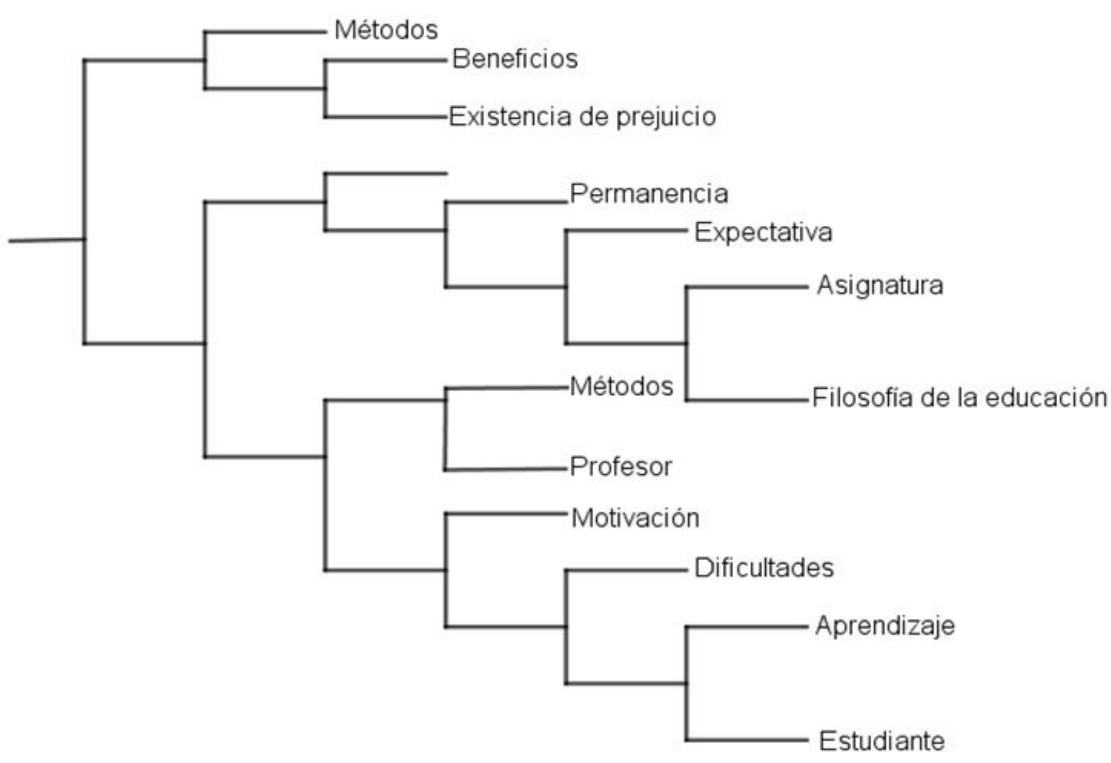

FIGURA 1

Conglomerados coeficiente de Jaccard

Nota: Esquema elaborado para la investigación con software Nvivo 11 Fuente: Elaboración propia

Entre los elementos relevantes que aparecen en el conglomerado está que el aprendizaje está asociado al estudiante, que la motivación del estudiantado está asociada a las dificultades del aprendizaje, que la asignatura de Filosofía de la Educación está lejana a los beneficios.

Posteriormente, se procede a efectuar una jerarquización de las categorías en que se establece una categoría inferior pero que luego evoluciona a una superior, lo cual permite construir una percepción del estudiantado, que se muestra en la Tabla 5. Se evidencia la argumentación para que una categoría quedara clasificada por encima o debajo de otra. 
TABLA 5

Jerarquía de categorías A

\begin{tabular}{|c|c|c|c|}
\hline Entrevista & Inferior & Justificaciones & Superior \\
\hline I y II & $\begin{array}{l}\text { Motivación } \\
\text { del y la } \\
\text { Estudiante }\end{array}$ & $\begin{array}{l}\text { La motivación de la } \\
\text { estudiante para asistir a la } \\
\text { asignatura es simplemente } \\
\text { por deber, obligación u } \\
\text { saber de otro área o tema. } \\
\text { Dificultad para con la } \\
\text { asignatura desmotiva a } \\
\text { aprender. Hay más } \\
\text { motivación de pasar por } \\
\text { deber u obligación }\end{array}$ & Aprendizaje \\
\hline Conclusión & \multicolumn{3}{|c|}{$\begin{array}{l}\text { La motivación adecuada leva hacia un objetivo bueno, } \\
\text { a saber, que el aprendizaje se produzca en el y la. } \\
\text { estudiante por ello tiene un nivel inferior }\end{array}$} \\
\hline \multicolumn{4}{|c|}{ Jerarquía de categorías $\mathrm{B}$} \\
\hline Entrevista & Inferior & Justificaciones & Superior \\
\hline I y II & Expectativa & $\begin{array}{l}\text { Expresa que espera un } \\
\text { carácter más dinámico de } \\
\text { la asignatura. Expresa que } \\
\text { espera un carácter más } \\
\text { dinámico de la asignatura } \\
\text { y que ello les ayuda a } \\
\text { facilitar el aprendizaje }\end{array}$ & $\begin{array}{l}\text { Asignatura } \\
\text { Filosófica }\end{array}$ \\
\hline Conclusión & \multicolumn{3}{|c|}{$\begin{array}{l}\text { La esperanza de los y las estudiantes gira en torno a la } \\
\text { asignatura filosófica, por eso la expectativa está en un } \\
\text { nivel inferior }\end{array}$} \\
\hline \multicolumn{4}{|c|}{ Jerarquía de categorías C } \\
\hline Entrevista & Inferior & Justificaciones & Superior \\
\hline I y II & $\begin{array}{l}\text { Método del } \\
\text { o la } \\
\text { Profesor(a) }\end{array}$ & $\begin{array}{l}\text { Hace hincapié que el o la } \\
\text { profesor(a) tiene una } \\
\text { gestión didáctica en el } \\
\text { desarrollo de las clases se } \\
\text { expresa que el } \\
\text { desenvolvimiento de la } \\
\text { gestión didáctica del } \\
\text { profesor(a) es deficiente }\end{array}$ & Profesor(a) \\
\hline Conclusión & \multicolumn{3}{|c|}{$\begin{array}{l}\text { El método es una acción del o la Profesor(a), por ende, } \\
\text { tiene un nivel inferior }\end{array}$} \\
\hline \multicolumn{4}{|c|}{ Jerarquía de categorias D } \\
\hline Entrevista & Inferior & Justificaciones & Superior \\
\hline I & Beneficio & $\begin{array}{l}\text { Expresa que la asignatura } \\
\text { tiene una importancia } \\
\text { para los y las estudiantes } \\
\text { que cursan la carrera de } \\
\text { Pedagogia en la Educación } \\
\text { Diferencial, es decir, ser } \\
\text { un profesional mejor. }\end{array}$ & $\begin{array}{l}\text { Asignatura } \\
\text { Filosófica }\end{array}$ \\
\hline Conclusión & \multicolumn{3}{|c|}{$\begin{array}{l}\text { El beneficio es un efecto de la asignatura por ello tiene } \\
\text { un nivel inferior }\end{array}$} \\
\hline
\end{tabular}

Fuente: Elaboración propia

De todo este proceso, se procede a ejecutar una jerarquización final de las categorías establecidas que se observa en la Figura 2, y muestra el resultado final de la jerarquización que se obtuvo del análisis de las entrevistas al estudiantado de pedagogía. 


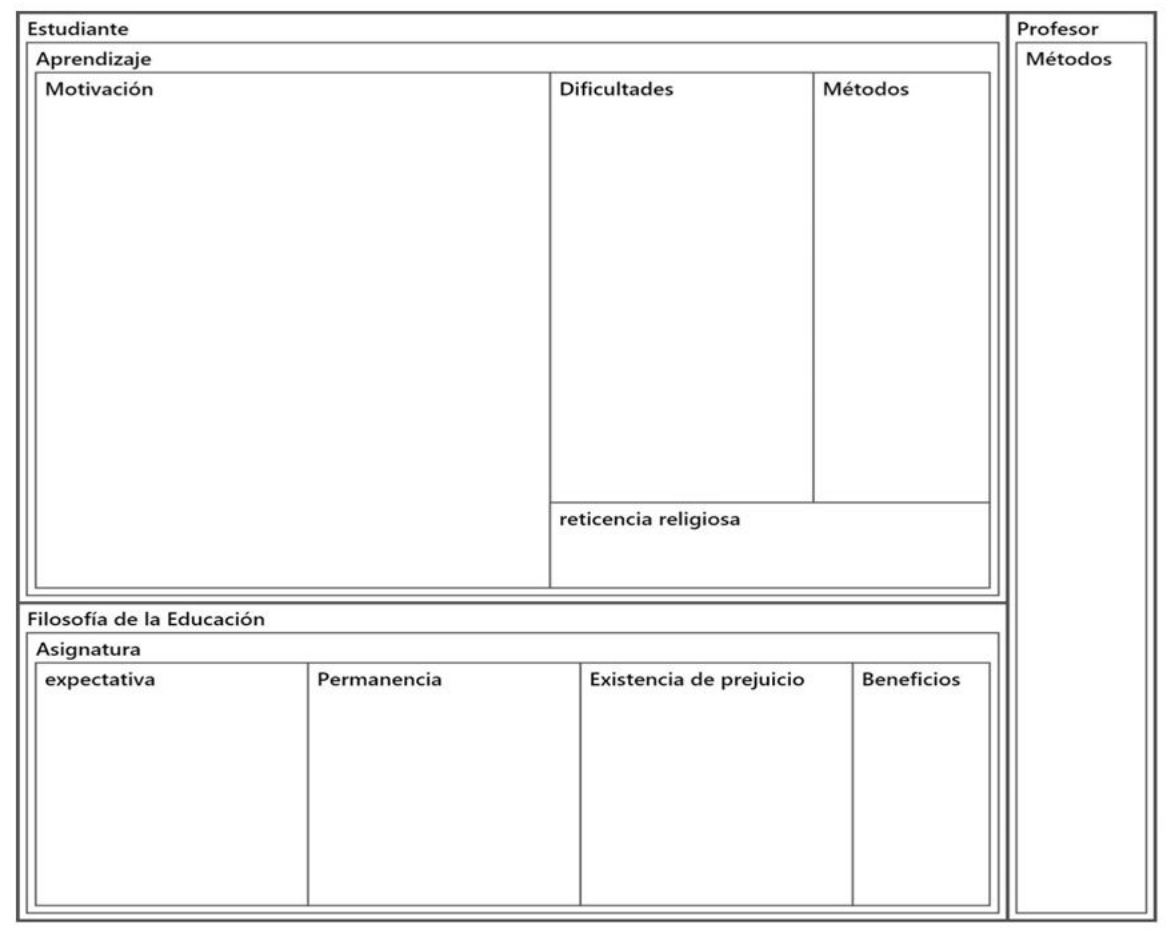

FIGURA 2

Jerarquía total de categorías

Nota: Esquema elaborado para la investigación con software Nvivo 11

Fuente: Elaboración propia

De esta jerarquización se vislumbra que la motivación tiene una notoria incidencia para el aprendizaje filosófico que se espera de la asignatura Filosofía de la Educación, seguido por las dificultades que se le presentan para ello y los métodos utilizados por el estudiantado; también le sigue en cuanto notoriedad los métodos empleados por el personal docente.

Luego de este proceso se levanta un mapa, que se observa en la Figura 3, del contenido presente en las entrevistas seleccionadas que muestra el proceso de cómo se llegó al análisis cualitativo a partir de la información obtenida, y en donde los temas son: el profesor(a) -y los métodos que utiliza-; la motivación para la Filosofía desde la perspectiva del estudiantado -en donde las dificultades, métodos y reticencia religiosa son factores que inciden-; y la Filosofía en cuanto asignatura, hay existencia de prejuicios, pero también expectativas, hay interés por la permanencia del curso filosófico y reconocimiento de los beneficios que tiene. 


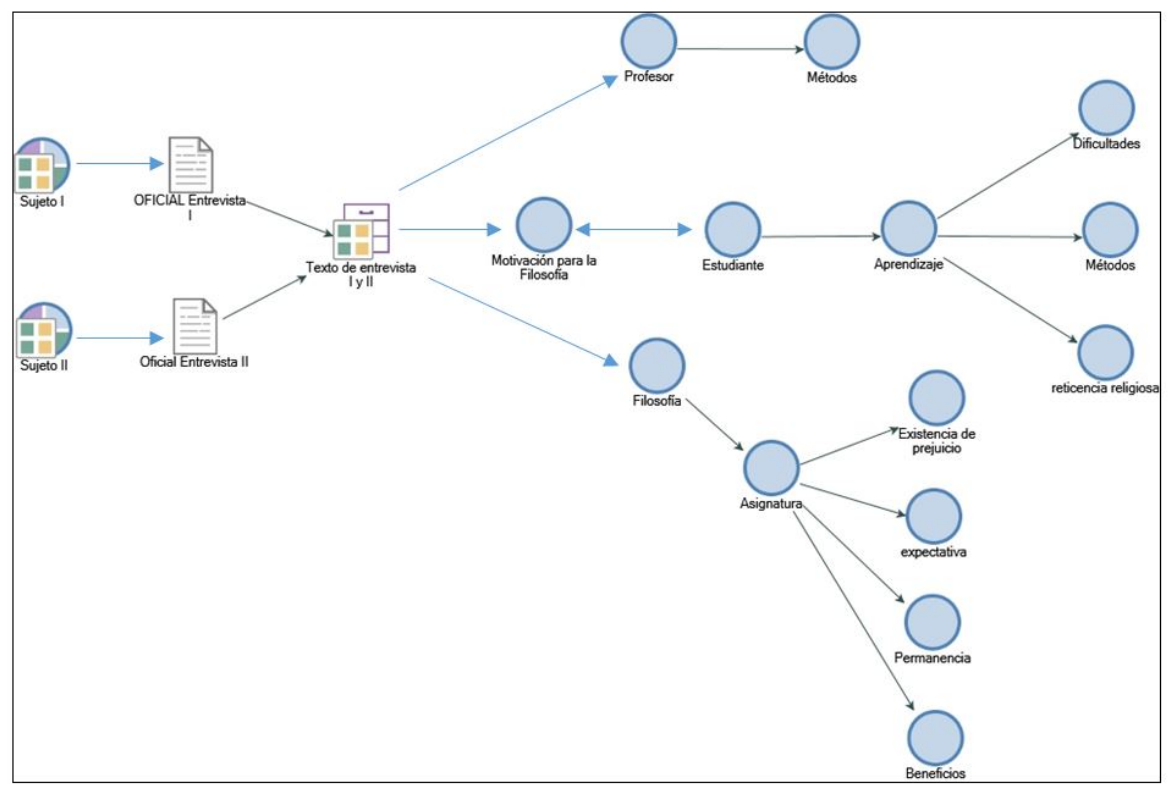

FIGURA 3

Mapa de contenido de las entrevistas

Nota: Esquema elaborado para la investigación con software Nvivo 11

Fuente: Elaboración propia

Así, a partir del mapa, se puede señalar que la observación más relevante dice relación con docente, en cuanto a los métodos utilizados en su docencia, con respecto a la motivación del estudiantado para con la Filosofía, en donde ellos con respecto al aprendizaje tienen sus propios métodos, tienen algunas dificultades para el aprendizaje y cierta reticencia religiosa. Por último, se encuentra el tema de la asignatura filosófica en el que, si bien hay prejuicios, el estudiantado ratifica la permanencia de la asignatura dentro de la malla curricular; tiene expectativas y ven beneficios.

A partir de todos estos elementos se procede a efectuar la interpretación de los datos obtenidos.

\section{INTERPRETACIÓN DE LOS RESULTADOS}

Los resultados de este trabajo permiten señalar que se expresa la existencia de una situación de motivación disminuida por parte del estudiantado que participa en la asignatura Filosofía de la Educación, por ende esto tendría un impacto en los logros de los objetivos de aprendizaje en la asignatura filosófica. Cabe precisar, que al existir una disminuida motivación personal, el alumnado opta por pasar la asignatura de filosofía simplemente por conveniencia. Además, para este tiene una pobre vinculación con la formación académica pedagógica. Estos elementos llevan a que en el mapa de análisis la motivación sea un factor muy potente; en este aspecto hay una validación de lo afirmado por López (2009) en lo referente a la importancia de la motivación en el ámbito educativo.

Se afirma que esta asignatura debe estar en la malla académica de la carrera, eso sí es conveniente que tenga una mayor referencia o pertinencia al objeto de la pedagogía, pues se observa un quiebre en la relación esperada entre teoría y praxis. Esta relación está en sintonía con lo manifestado por el autor Álvarez (2012).

Se expresa que la asignatura filosófica es un apoyo para quien lo quisiera utilizar y ayuda a ser un(a) mejor profesor(a). Este dato expresa la veracidad sobre la importancia de la Filosofía para el ser humano lo que es manifestado por distintos autores como por ejemplo Kohan (2010).

Se explica que se debe perfeccionar los recursos didácticos utilizados por el profesorado en el proceso de enseñanza de la asignatura Filosofía de la Educación, dado que es una ayuda importante para mantener una 
buena expectativa del estudiantado. En esta información hay una correlación importante con los autores que afirman la necesidad de potenciar una didáctica específica para la Filosofía, por ejemplo, Cerletti (2008).

Los métodos de aprendizaje que empleará el estudiantado para el desarrollo de la asignatura respectiva son, de modo individual, la lectura de textos, responder a las preguntas que hace el cuerpo docente, desarrollar trabajos prácticos; y de modo grupal, se destaca la lectura y compresión de textos, al igual que hacer resúmenes, conversación entre las alumnas y la reproducción de videos que favorezcan un análisis filosófico. La importancia de que ellas utilicen estos elementos es, por un lado, porque efectivamente les ayuda en su proceso de aprendizaje para la asignatura de filosofía (López A., 2009); y por otro, se entregan lineamientos que favorecen la creación de estrategias de enseñanza nuevas o potenciar las que ya existen para ser aplicadas durante el desarrollo de las clases de Filosofía educativa (Cerletti, 2008).

\section{CoNCLUSIÓN}

Considerando la información entregada por el estudiantado de pedagogía con su respectiva interpretación y el objetivo de este estudio, se puede expresar que la percepción que tienen sobre la Filosofía de la Educación manifiesta la existencia de un problema motivacional hacia esta asignatura, y a la Filosofía en general, encontrándose una sintonía con los planteamientos de autores como López (2009) y Muñoz (2015), quienes ya se han referido en estas páginas; además, se ratifica la importancia de la presencia filosófica en el proyecto curricular y la necesidad de perfeccionar los recursos didácticos que utiliza el docente; esta opinión viene a reflejar una realidad que se ha venido dando hace un tiempo y por ello, es posible encontrar investigados, entre ellos algunos filósofos citados en este estudio, tales como: Moreno (2013), Cerletti (2008) y Azar (2015) que validan la afirmación hecha por el estudiantado, tal como se ha expresado en estas páginas. Junto a ello, también se encuentra un escaso conocimiento previo de parte del alumnado hacia las asignaturas de filosofía, en especial sobre la Filosofía de la Educación, lo cual muestra una baja conceptualización de esta disciplina, esta afirmación ya es consecuencia de situaciones que han ocurrido en el acontecer educacional de Chile, esto se vislumbraba a partir de lo expresado por Olivares (2015), en virtud del intento de sacar la Filosofía de la educación chilena.

El estudiantado evidencia en su percepción una falta de pertinencia de la asignatura filosófica con la carrera de pedagogía, ello conllevaría a revisar la necesidad de elaborar alguna actualización con respecto al programa de la asignatura. Otro punto por considerar, es que el personal docente debe tener estrategias metodológicas y didácticas pertinentes para el desarrollo de esta asignatura filosófica. En suma, a partir del logro del objetivo de este trabajo, a saber, dar cuenta y analizar cualitativamente la percepción que tienen los estudiantes de pedagogía sobre la Filosofía de la Educación, se afirma -por una parte- la necesidad de actualizar las estrategias didácticas sobre su enseñanza para que ayuden de modo concreto a estudiantes de pedagogía a desarrollar habilidades reflexivas sobre la elaboración del saber pedagógico; -y por otra- la importancia de profundizar sobre la necesidad de tener una didáctica específica de la Filosofía para el área de la pedagogía, ayudando a lograr un aprendizaje más profundo y significativo en el estudiantado, es decir, que alcancen a reflexionar adecuadamente sobre cómo lograr un adecuado saber pedagógico.

\section{ReFERENCIAS}

Altarejos, F. y Naval, C. (2011). Filosofía de la Educación. Pamplona, España: Ediciones Universidad de Navarra, S.A. Álvarez Á., C. (2012). ¿Qué sabemos de la relación entre la teoría y la práctica en la educación? Revista Iberoamericana de Educación, 60(2), 11.

Azar, R. (2015). ¿Qué sentido tiene enseñar y/o aprender filosofía? Revista de Filosofia Eikasia, 61(1), 189-198.

Bernal, L. (2015). Educación y Filosofía: un binomio olvidado por los nuevos modelos. Actas, 3(1), 1-13. 
Luis Rodrigo Camacho Verdugo, et al. Filosofía de la Educación y pedagogía de la enseñanza en la f...

Boavida, J. (2006). De una didáctica de la filosofía a una filosofía de la educación. Revista Española de Pedagogía 64(234), 205-226.

Camacho, L. R. (2015). La enseñanza desde la filosofía de Santo Tomás de Aquino (Pregrado). Universidad Católica de la Santísima Concepción, Concepción, Chile.

Camacho, L. R. (2017). Síntesis de la sistematización epistemológica de la Filosofía educativa. Revista de Filosofía, $16(1), 45-60$.

Campos A., C. C. (2012). Informe de Autoevaluación, Carrera Pedagogía en Educación Diferencial. Concepción de Chile: Universidad Católica de la Santísima Concepción.

Cárdenas, J. (2007). Metodologías de la enseñanza de la filosofía en la educación media chilena: un problema filosófico (Tesis de Pregrado). Universidad de Chile, Santiago, Chile

Carvajal, A. y García, J. (2004). ¿Cómo perciben los estudiantes universitarios la enseñanza de la Filosofía, según sus experiencias en la educación diversificada costarricense? Revista Electrónica Actualidades Investigativas en Educación, 4(1), 1-20.

Cerletti, A. (2008). La enseñanza de la filosofía como problema pedagógico. Buenos Aires: Editorial Zorzal.

Chávez, J. (2011). Filosofia de la Educación para maestros. La Habana, Cuba: Educación Cubana, Ministerio de Educación.

Cortés, G. (2015). Sobre la necesidad de la enseñanza de la Filosofía. Actas, 3(1), 193-216.

Donda, C. S. (2015). La enseñanza de la filosofía en medios académicos y extra-académicos. En A. Cerletti (Ed.), $L a$ enseñanza de la filosofía en perspectiva (pp. 140-151). Buenos Aires: Eudeba.

Ferrer, C. (27 de febrero de 2018). El futuro de Filosofía: Intelectuales y artistas buscan revertir la idea de eliminar la asignatura. El Mercurio, pp. 1-11.

Fullat, O. (1992). Filosofias de la Educación "PAIDEIA". Barcelona: CEAC.

García, C. H., y Monzón, M. (2015). Enseñanza de la Filosofía y Filosofía de la Educación. Actas, 3(1), 1-14.

González, A. (1963): Filosofia de la educación. Buenos Aires: Troquel.

Kohan, W. (2010).Filosofia, la paradoja de aprender y enseñar. Buenos Aires: Zorzal.

Lamoureux, A. (2006). Recherche et méthodologie en sciences humaines. Quebec: Beauchemin.

López A., M.-S. F., E. (2009). Estilos de aprendizaje. Relación con motivación y estrategias. Journal of Learning Style, University Utah Valley, 2(4), 36-55.

Martínez, E. (2011). Ser y educar: Fundamentos de Pedagogía Tomista. Bogotá, Colombia: Universidad Santo Tomás.

Millán, A. (2013). Obras Completas, Vol. II: Fundamentos de Filosofía. Madrid: Rialp.

Moreno, A. (2013). Percepciones de los estudiantes hacia la clase de Filosofía general en el campus central de la Universidad Nacional Autónoma de Honduras. Revista Ciencia y Tecnología, 12(1), 27-42.

Muñoz, M.-R., E. (2015). Desplazamiento de la filosofía de planes y programas en las instituciones formadoras de docentes: caso escuelas normales de México. Actas, 3(1), 1-16.

Olivares, R. (2015). La Filosofía secuestrada. Psicología y ética en la enseñanza de la filosofía en la educación chilena. Actas, 3(1), 1-16.

Rodríguez, G., Gil, J., y García, E. (1999). Metodología de la investigación cualitativa. Málaga: Aljibe.

Sarbach, A. (2005). ¿Quépasa en la clase de Filosofía?: Hacia una didáctica narrativa y de investigación (Tesis doctoral). Universidad de Barcelona, Barcelona.

UCSC (2016). Modelo Educativo de la Universidad Católica de la Santísima Concepción. Concepción, Chile: Ed. UCSC.

UNESCO. (2011). La Filosofía Una escuela de la Libertad. México: La Organización de las Naciones Unidas para la Educación, la Ciencia y la Cultura y Universidad Autónoma Metropolitana.

Vásquez, S. M. (2012). Filosofía de la Educación: estado de la cuestión y líneas esenciales. Buenos Aires: CIAFIC ediciones. 
Revista Educación, 2020, 44(1), Enero-Junio, ISSN: 0379-7082 / 2215-2644

CC BY-NC-ND 